\title{
Beam-Target Spin Asymmetries in Exclusive Pion Photoproduction of Deuteron
}

\author{
Suleiman S. Al-Thoyaib \\ Physics Department, Faculty of Science, Al-Qassim University, \\ Oyoun Al-Jawa, Saudi Arabia \\ salthoyaib@yahoo.com
}

\begin{abstract}
Beam-target double polarization asymmetries in the exclusive pion photoproduction reaction from the deuteron is investigated at photon energies close to threshold and near the $\Delta(1232)$-resonance with inclusion of nucleon-nucleon and pionnucleon final state interaction effects. The elementary pion photoproduction amplitude from the free nucleon is taken from the unitary isobar MAID-2003 model. The interactions in the final nucleon-nucleon and pion-nucleon subsystems are taken in separable form. The role of final-state interaction effects on double spin asymmetries for circularly polarized photon and both vector and tensor polarized deuteron are found to be important. It must be taken into consideration in the analysis of future experimental measurements.
\end{abstract}

Keywords: Polarization phenomena in reactions; Photoproduction reactions; Final-state interactions.

\section{Introduction}

Since the advent of high duty-factor accelerators, such as MAMI in Mainz and ELSA in Bonn (Germany) or Jefferson-Lab in Newport News and LEGS in Brookhaven (USA), the study of single pion production reaction in intermediate energy nuclear physics has been getting more and more attention in recent years ${ }^{[1-6]}$. The main goal of this study is to investigate the structure of hadrons in the non-perturbative domain of Quantum Chromo-dynamics (QCD) and therefore the nature of strong interactions. 
Single and double polarization observables for the inclusive singlepion photoproduction reaction from the deuteron have been studied by several authors. In Ref. [2,3], special emphasis is given for the beamtarget double spin asymmetry and the Gerasimov-Drell-Hearn (GDH) sum rule. Single- and double-spin asymmetries for pion photoproduction from the deuteron have been predicted in Ref. [7-9] without any kind of FSI effects. Arenhoevel and Fix ${ }^{[4]}$ as well as Levchuk et al. ${ }^{[5]}$ have been studied the same reaction with particular emphasis to the extension to higher energies. Most recently, Darwish et al. ${ }^{[6]}$ have investigated the inclusive pion photoproduction reaction from the deuteron with polarized photon beam and polarized deuteron target including FSI effects. They have studied the influence of various elementary amplitudes on observables and found that the $\mathrm{d}(\gamma, \pi) \mathrm{NN}$ reaction may serve as a filter for elementary reactions. Unfortunately, all these approaches were restricted to the inclusive reaction $\mathrm{d}(\gamma, \pi) \mathrm{NN}$. However, the exclusive reaction $\mathrm{d}(\gamma, \pi \mathrm{N}) \mathrm{N}$, where both the pion and the nucleon are observed in coincidence, is also of great importance, because of the planned experimental measurements at LEGS in Brookhaven National Laboratory.

Therefore, the main goal of this paper is to extend our previous work $^{[10]}$, in which theoretical predictions for the doubly polarized total and differential cross sections for the exclusive reaction $\mathrm{d}(\gamma, \pi \mathrm{N}) \mathrm{N}$ were given, to investigate the influence of FSI effects on the beam-target double polarization observables in the photon lab-energies close to pion threshold and near the $\Delta(1232)$-resonance. These additional observables contain interference terms of the various amplitudes in different combinations from which one can determine all amplitudes provided one has measured a complete set of observables. The calculation is of high theoretical interest, because it provides an important test of our understanding of the $\pi \mathrm{NN}$ dynamics. That understanding is a prerequisite for reliable extraction of the pion photoproduction amplitude on the neutron.

In the next section, we briefly review the model which we use in our calculations ${ }^{[1]}$. The results of the formalism to calculate the beamtarget double polarization asymmetries are presented and discussed in Section 3 . 


\section{Theoretical model}

Following the formulation of the previous work $^{[1]}$, the unpolarized differential cross section for pion photoproduction from the deuteron is given by

$$
\frac{d \sigma}{d \Omega_{\pi}}=\frac{1}{6} \int_{0}^{q_{\max }} d q \int d \Omega_{p_{N N}} \rho_{s} \sum_{s m t m_{\gamma} m_{d}}\left|\mathcal{M}_{s m m_{\gamma} m_{d}}^{(t \mu)}\right|^{2} .
$$

All observables are determined by the photoproduction amplitude $\mathcal{M}_{s m m_{\gamma} m_{d}}^{(t \mu)}$. In principle, the full treatment of all interaction effects requires a full unitary $\pi \mathrm{NN}$ three-body calculation. However, the treatment of the scattering amplitude in this work is completely analogous to previous work $^{[1]}$. The transition M-matrix elements are calculated in the frame of time-ordered perturbation theory, using the elementary pion photoproduction operator introduced in Ref. [12] and including $\mathrm{NN}$ - and $\pi \mathrm{N}$-rescattering in the final state. The amplitude of the $\gamma \mathrm{d} \rightarrow \pi \mathrm{NN}$ reaction is determined by the transition matrix

$$
\mathcal{M}_{s m m_{\gamma} m_{d}}^{(t \mu)}={ }^{(-)}\left\langle\vec{q} \mu, \overrightarrow{p_{1}} \overrightarrow{p_{2}} s m t-\mu\left|\epsilon_{\mu}\left(m_{\gamma}\right) J^{\mu}(0)\right| \vec{d} m_{d} 00\right\rangle
$$

where $\mathrm{J}^{\mathrm{\mu}}(0)$ denotes the current operator. The total transition matrix element reads in this work

$$
\mathcal{M}_{s m m_{\gamma} m_{d}}^{(t \mu)}=\mathcal{M}_{s m m_{\gamma} m_{d}}^{(t \mu) I A}+\mathcal{M}_{s m_{\gamma} m_{d}}^{(t \mu) N N}+\mathcal{M}_{s m m_{\gamma} m_{d}}^{(t \mu) \pi N}
$$

where the first term denotes the transition matrix of the plane wave impulse approximation (IA) which means that the reaction will take place only on one of the nucleons leaving the other as a pure spectator. The second term represents the corresponding matrix for the NN-rescattering, while the third one denotes the rescattering contribution from $\pi \mathrm{N}$ rescattering.

Denoting the matrix element of the elementary production on a nucleon by $\hat{t}_{\gamma \pi}^{N}$, the amplitude of the IA-term in the deuteron lab-frame reads $^{[1]}$ 


$$
\begin{gathered}
\mathcal{M}_{s m m_{\gamma} m_{d}}^{(t \mu) I A}\left(\vec{k}, \vec{q}, \vec{p}_{1}, \vec{p}_{2}\right)=\sqrt{2} \sum_{m^{\prime}}\langle s m, t-\mu|\left(\left\langle\vec{p}_{1}\left|\hat{t}_{\gamma \pi}^{N}(\vec{k}, \vec{q})\right|-\vec{p}_{2}\right\rangle \tilde{\Psi}_{m^{\prime}, m_{d}}\left(\vec{p}_{2}\right)\right. \\
\left.-(-)^{s+t}\left(\vec{p}_{1} \leftrightarrow \vec{p}_{2}\right)\right)\left|1 m^{\prime}, 00\right\rangle,
\end{gathered}
$$

where $\mid 1 \mathrm{~m}^{\prime}, 00>$ denotes the two-nucleon spin and isospin wave function.

By using the information in Ref. [1], we can write the NNrescattering term in (3) as

$$
\begin{aligned}
\mathcal{M}_{s m m_{\gamma} m_{d}}^{(t \mu) N N}\left(\vec{k}, \vec{q}, \vec{p}_{1}, \vec{p}_{2}\right)= & \sum_{m^{\prime}} \int d^{3} \vec{p}_{N N}^{\prime} \sqrt{\frac{E_{1} E_{2}}{E_{1}^{\prime} E_{2}^{\prime}}} \widetilde{\mathcal{R}}_{s m m^{\prime}}^{N N, t \mu}\left(W_{N N}, \vec{p}_{N N}, \vec{p}_{N N}^{\prime}\right) \\
& \times \frac{M_{N}}{\tilde{p}^{2}-p_{N N}^{\prime 2}+i \epsilon} \mathcal{M}_{s m^{\prime}, m_{\gamma} m_{d}}^{(t \mu) I A}\left(\vec{k}, \vec{q}, \vec{p}_{1}^{\prime}, \vec{p}_{2}^{\prime}\right)
\end{aligned}
$$

The conventional NN-scattering matrix $\widetilde{\mathcal{R}}_{s m m^{\prime}}^{N N, t \mu}$ is introduced with respect to noncovariantly normalized states. It is expanded in terms of the

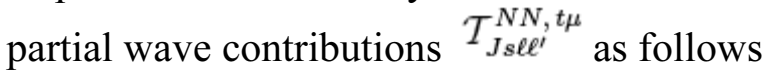

$$
\tilde{\mathcal{R}}_{s m m^{\prime}}^{N N, t \mu}\left(W_{N N}, \vec{p}_{N N}, \vec{p}_{N N}^{\prime}\right)=\sum_{J \ell \ell^{\prime}} \mathcal{F}_{\ell \ell^{\prime} m m^{\prime}}^{N N, J s}\left(\hat{p}_{N N}, \hat{p}_{N N}^{\prime}\right) \mathcal{T}_{J s \ell \ell^{\prime}}^{N N, t \mu}\left(W_{N N}, p_{N N}, p_{N N}^{\prime}\right),
$$

where the purely angular function $\mathcal{F}_{\ell \ell^{\prime} m m^{\prime}}^{N N, J s}\left(\hat{p}_{N N}, \hat{p}_{N N}^{\prime}\right)$ is defined by

$$
\mathcal{F}_{\ell \ell^{\prime} m m^{\prime}}^{N N, J s}\left(\hat{p}_{N N}, \hat{p}_{N N}^{\prime}\right)=\sum_{M m_{\ell} m_{\ell^{\prime}}} C_{m_{\ell} m M}^{\ell s J} C_{m_{\ell^{\prime}} m^{\prime} M}^{\ell^{\prime} s J} Y_{\ell m_{\ell}}^{\star}\left(\hat{p}_{N N}\right) Y_{\ell^{\prime} m_{\ell^{\prime}}}\left(\hat{p}_{N N}^{\prime}\right)
$$

The necessary half-off-shell NN-scattering matrix ${ }^{\mathcal{T}_{J s \ell \ell^{\prime}}^{N N, t \mu} \text { was }}$ obtained from separable representation of a realistic NN-interaction ${ }^{[13]}$. Explicitly, all S, P, and D waves were included in the NN-scattering matrix.

The transition matrix element for the $\pi \mathrm{N}$-rescattering in the final state has formally a similar structure as the one for NN-rescattering and is given by ${ }^{[1]}$ 


$$
\begin{aligned}
\mathcal{M}_{s m m_{\gamma} m_{d}}^{(t \mu) \pi N}\left(\vec{k}, \vec{q}, \vec{p}_{1}, \vec{p}_{2}\right)= & \frac{1}{2} \sum_{s^{\prime} m^{\prime} t^{\prime} \mu^{\prime}} \int d^{3} \vec{p}_{2}^{\prime} \sqrt{\frac{\omega_{q} E_{2}}{\omega_{q^{\prime}} E_{2}^{\prime}}} \sum_{m_{2} m_{2}^{\prime} \mu_{2} \mu_{2}^{\prime} \tilde{\mu} \tilde{\mu}} \mathcal{C}_{\alpha \alpha^{\prime}}^{\tilde{t} \tilde{\mu}}\left(m_{2}, m_{2}^{\prime}, \mu_{2}, \mu_{2}^{\prime}\right) \\
& \times\left[\sum_{J \ell} \mathcal{F}_{J \ell m_{2} m_{2}^{\prime}}^{\pi N}\left(\hat{p}_{\pi N}, \hat{p}_{\pi N}^{\prime}\right) \mathcal{T}_{J \ell}^{\pi N, \tilde{t} \tilde{\mu}}\left(W_{\pi N}\left(\vec{p}_{2}\right), p_{\pi N}, p_{\pi N}^{\prime}\right)\right. \\
& \times \mathcal{G}_{0}^{\pi N N(+)}\left(E_{\gamma d}, \vec{q}^{\prime}, \vec{p}_{1}, \vec{p}_{2}^{\prime}\right) \\
& \left.\times \mathcal{M}_{s^{\prime} m^{\prime}, m_{\gamma} m_{d}}^{\left(t^{\prime} \mu^{\prime}\right) I A}\left(\vec{k}, \vec{q}^{\prime}, \vec{p}_{1}, \vec{p}_{2}^{\prime}\right)-(-)^{s+t}\left(\vec{p}_{1} \leftrightarrow \vec{p}_{2}\right)\right]
\end{aligned}
$$

where we have defined

$$
\mathcal{F}_{J \ell m_{2} m_{2}^{\prime}}^{\pi N}\left(\hat{p}_{\pi N}, \hat{p}_{\pi N}^{\prime}\right)=\sum_{m_{\ell} m_{\ell}^{\prime} M} C_{m_{2} m_{\ell} M}^{\frac{1}{2} \ell J} C_{m_{2}^{\prime} m_{\ell}^{\prime} M}^{\frac{1}{2} \ell J} Y_{\ell m_{\ell}}^{\star}\left(\hat{p}_{\pi N}\right) Y_{\ell m_{\ell}^{\prime}}\left(\hat{p}_{\pi N}^{\prime}\right)
$$

The relative momentum of the final (initial) pion-nucleon subsystem is given, respectively, by

$$
\vec{p}_{\pi N}=\frac{M_{N} \vec{q}-m_{\pi} \vec{p}_{2}}{M_{N}+m_{\pi}}, \quad \vec{p}_{\pi N}^{\prime}=\frac{M_{N} \vec{q}^{\prime}-m_{\pi} \vec{p}_{2}^{\prime}}{M_{N}+m_{\pi}}=\frac{M_{N}}{M_{N}+m_{\pi}}\left(\vec{q}+\vec{p}_{2}\right)-\vec{p}_{2}^{\prime} .
$$

In the present work we calculate the various beam-target double polarization observables for the exclusive pion photoproduction reaction from the deuteron using the MAID-2003 model ${ }^{[12]}$. It is a unitary isobar model for investigating single pion photo- and electroproduction off protons and neutrons in the energy region up to $2 \mathrm{GeV}$. This model is based on a non-resonant background described by Born terms and vector meson exchange contributions and nucleon resonance excitations modeled by Breit-Wigner functions

$$
t_{\gamma \pi}=t_{\gamma \pi}^{B}+t_{\gamma \pi}^{R} .
$$

Both parts, background and resonance are separately unitarized for each partial wave up to the $2 \pi$ threshold. The Feynman diagrams for these terms are shown in Fig. 1.

For the nucleon-nucleon and pion-nucleon scattering in the NN- and $\pi \mathrm{N}$-subsystem, respectively, we use separable potentials from Ref. [13] for $\mathrm{NN}$ and Ref. [14] for $\pi \mathrm{N}$. These separable potentials have played a major role in the development of few-body physics and widely used in case of the $\pi \mathrm{NN}$ system (see for example Ref. [15] and references therein). Therefore, for the present study of the influence of $\mathrm{NN}$ - and $\pi \mathrm{N}$ rescattering these models are good enough.

With respect to polarization observables, we consider in this paper the beam-target double polarization observables for circularly polarized 
photons and both vector and tensor polarized deuteron $\mathrm{T}_{10}{ }^{\text {circ }}, \mathrm{T}_{11}{ }^{\text {circ }}$, $\mathrm{T}_{21}{ }^{\text {circ }}$ and $\mathrm{T}_{22}{ }^{\text {circ }}$. The explicit expressions for these polarization observables are given in Ref. [4].

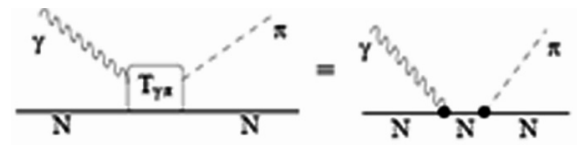

(A)

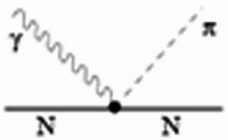

(D)

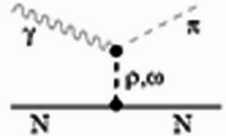

(E)

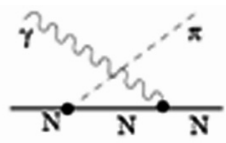

(B)

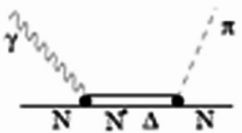

(F)

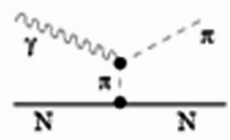

(C)

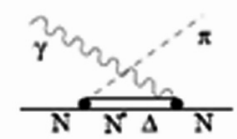

(G)

Fig. 1. Feynman diagrams for pion photoproduction from the nucleon. Born terms: (A) direct nucleon pole, (B) crossed nucleon pole, (C) pion in flight, and (D) KrollRudermann contact term; (E) vector-meson exchange ( $\rho$ and $\omega)$; resonance excitations contribution: (F) direct and (G) crossed.

\section{Results and Discussion}

Here we present and discuss the results we have predicted for the beam-target double polarization observables for circularly polarized photons and both vector and tensor polarized deuteron $\mathrm{T}_{10}{ }^{\text {circ }}, \mathrm{T}_{11}{ }^{\text {circ }}$, $\mathrm{T}_{21}{ }^{\text {circ }}$, and $\mathrm{T}_{22}{ }^{\text {circ }}$. As mentioned above, for the calculation of the NNrescattering contribution we have taken the separable representation of the realistic Paris potential from Ref. [13] and included all partial waves up to ${ }^{3} \mathrm{D}_{3}$. Also, the deuteron wave function was calculated using this potential. Similarly, $\pi \mathrm{N}$-rescattering is evaluated using a realistic separable representation of the $\pi \mathrm{N}$-interaction from Ref. [14] and taking into account all partial waves up to $1=2$. We have evaluated the various polarization asymmetries in pure plane wave impulse approximation alone and with inclusion of $\mathrm{NN}$ - and $\pi \mathrm{N}$-rescattering. As already mentioned, the elementary pion photoproduction amplitude from free nucleons is taken from the MAID-2003 model ${ }^{[12]}$.

As first, we would like to mention that the results we have obtained for the two charged pion channels are in general quite similar. Therefore, we display here the results only for the negative pion channel in addition to the neutral one.

We begin the discussion of the results with the double spin asymmetries for circularly polarized real photons and vector polarized deuteron, i.e., $\mathrm{T}_{10}{ }^{\text {circ }}$ and $\mathrm{T}_{11}{ }^{\text {circ }}$, as shown in Fig. 2 (for neutral-pion 
channel) and Fig. 3 (for negative-pion channel), respectively. In order to cover the energy range from threshold to the $\Delta(1232)$-resonance region, we present results at photon energies close to threshold $\left(\mathrm{E}_{\gamma}=155 \mathrm{MeV}\right)$ and near the $\Delta(1232)$-resonance $\left(\mathrm{E}_{\gamma}=320 \mathrm{MeV}\right)$.

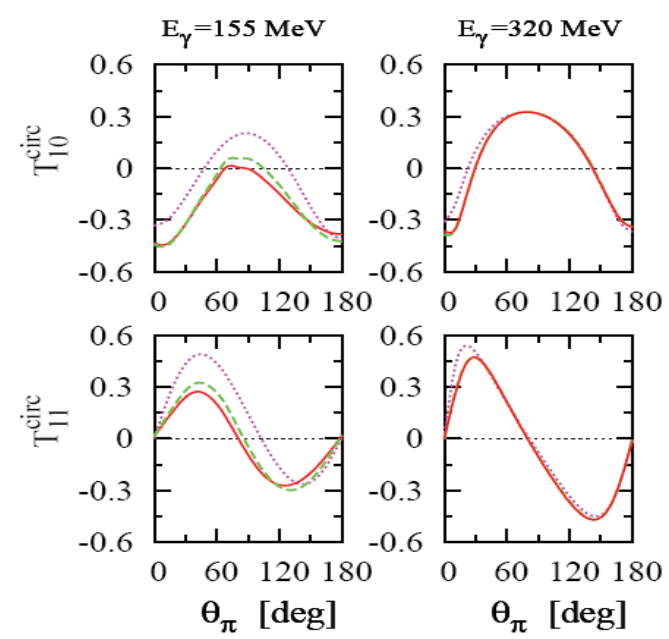

Fig. 2. The beam-target double polarization observables $T_{10}{ }^{\text {cire }}$ and $T_{11}{ }^{\text {cire }}$ for circularly polarized photons and vector polarized deuterons of exclusive $\pi^{0}$-photoproduction from the deuteron as a function of pion angle in the laboratory frame of the deuteron at two different photon lab-energies using the elementary pion photoproduction operator from MAID-2003 [12] and including FSI effects. The dotted, dashed, and solid curves represent the results of IA, IA+NN, and $\mathrm{IA}+\mathrm{NN}+\pi \mathrm{N}$, respectively.
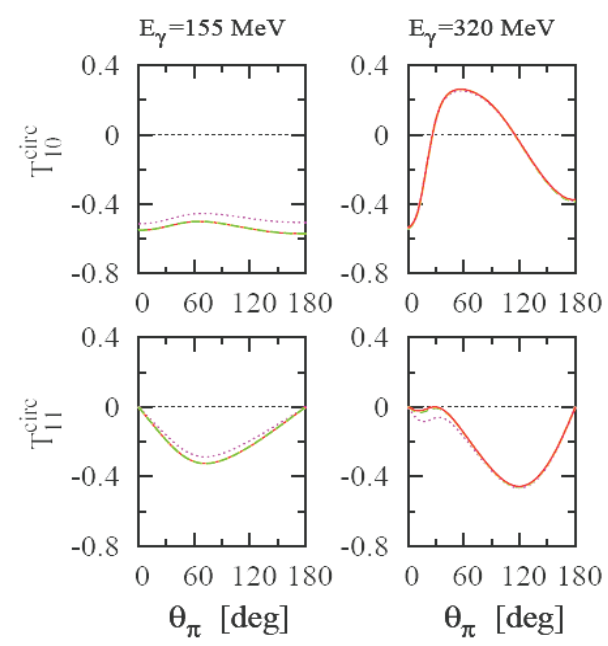

Fig. 3. Same as in Fig. 2 but for the exclusive negative-pion photoproduction channel. 
One readily notes that a significant role of the nucleon-nucleon and pion-nucleon rescattering in exclusive pion photoproduction from the deuteron at very low energies is seen in both $\mathrm{T}_{10}$ circ and $\mathrm{T}_{11}{ }^{\text {circ }}$ asymmetries (see the left two panels in Fig. 2). This contribution becomes very high at the peak position, while it is small at extreme backward pion angles. When we go to higher energies near the $\Delta(1232)$ resonance, one sees that the significant contribution from rescattering effects becomes very small and completely negligible at pion angles greater than $50^{\circ}$. We would like to point out that the $\mathrm{T}_{10}{ }^{\text {circ }}$ asymmetry is of great importance because of its use to determine the Gerasimov-DrellHearn $(\mathrm{GDH})$ sum rule ${ }^{[16]}$.

For charged pion channels (see Fig. 3 for negative-pion production), one readily notes that the contribution from final state interaction effects are in general quite small even at photon energies close to threshold. Only at $320 \mathrm{MeV}$, one notes for the $\mathrm{T}_{11}{ }^{\text {circ }}$ asymmetry a contribution to nucleon-nucleon rescattering at extreme forward pion angles. The main effect comes from the nucleon-nucleon rescattering contribution, while pion-nucleon rescattering is almost negligible. The $\mathrm{T}_{11}{ }^{\text {circ }}$ asymmetry is shown to be negative for negative-pion channel, while for neutral-pion production it has a positive maximum around $30^{\circ}$ and a negative maximum around $150^{\circ}$.

Next, we turn to the double polarization asymmetries for circularly polarized real photons and tensor polarized deuteron, i.e., the asymmetries $\mathrm{T}_{21}{ }^{\text {circ }}$ and $\mathrm{T}_{22}{ }^{\text {circ }}$, as shown in Fig. 4 (for neutral-pion channel) and Fig. 5 (for negative-pion channel). In contrast to the double polarization asymmetries for circularly polarized photon and vector polarized deuteron (Fig. 2), one notes here a fully different behavior for the asymmetries. In case of neutral pion channel (see Fig. 4, one notes that both nucleon-nucleon and pion-nucleon rescattering contribute. Their contribution is noticeable at both energies for both asymmetries. It is also clear that the $\mathrm{T}_{22}{ }^{\text {circ }}$ asymmetry is small in comparison to $\mathrm{T}_{21}{ }^{\text {circ }}$, in particular at energies close to threshold.

For charged pion channels, one notes quite small final state interaction effects in contrast to neutral pion channel as displayed in Fig. 5. Both asymmetries here are small. The $\mathrm{T}_{22}{ }^{\text {circ }}$ and $\mathrm{T}_{21}{ }^{\text {circ }}$ asymmetries have a peak at extreme forward angles and energies near the $\Delta(1232)$ resonance. They begin positive at threshold and then becomes negative 
near the $\Delta(1232)$ region. These asymmetries are more sensitive to final state interaction effects.

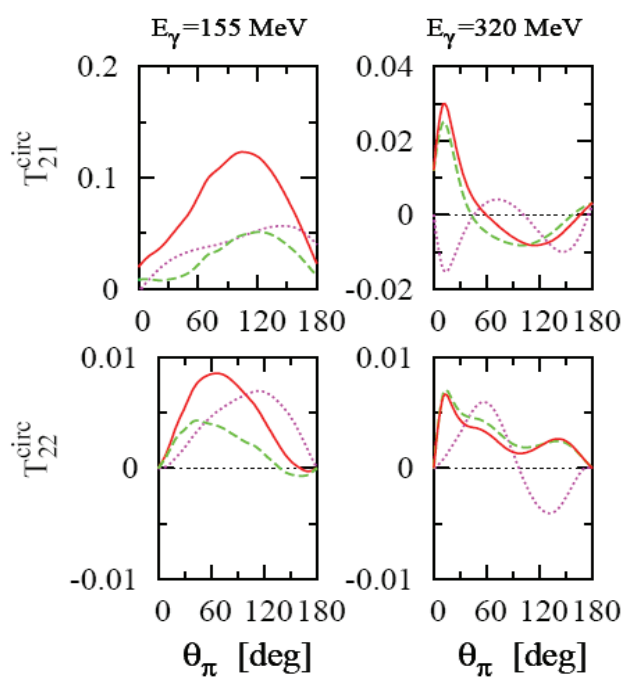

Fig. 4. The beam-target double polarization observables $T_{21}{ }^{\text {cire }}$ and $T_{22}{ }^{\text {circ }}$ for circularly polarized photons and tensor polarized deuterons of exclusive $\pi^{0}$-photoproduction from the deuteron as a function of pion angle in the laboratory frame of the deuteron at two different photon lab-energies using the elementary pion photoproduction operator from MAID-2003 ${ }^{[12]}$ and including FSI effects. The dotted, dashed, and solid curves represent the results of IA, IA+NN, and $\mathrm{IA}+\mathrm{NN}+\pi \mathrm{N}$, respectively.
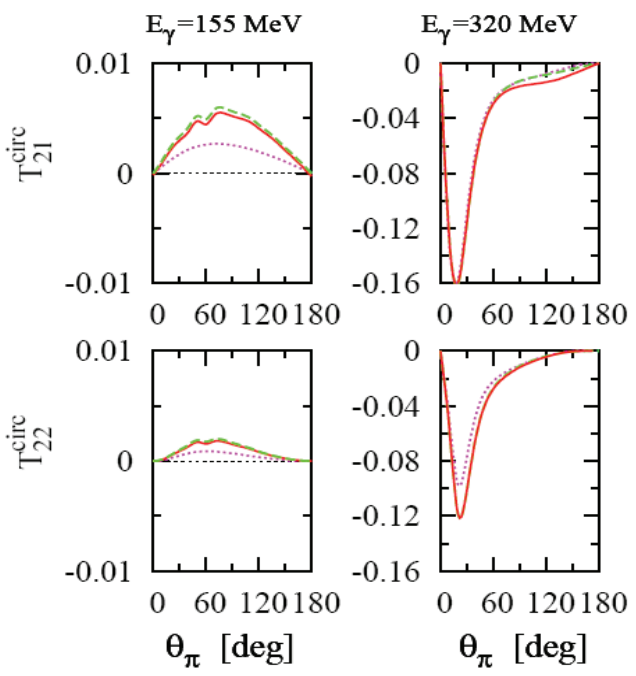

Fig. 5. Same as in Fig. 4 but for the exclusive negative-pion photoproduction channel. 
In summary, in this work we have explored the influence of beamtarget double polarization asymmetries in the exclusive pion photoproduction from the deuteron with particular emphasis on the influence of final state interaction in the nucleon-nucleon and pionnucleon subsystems of the final state. We have considered here the beamtarget double polarization observables for circularly polarized photons and both vector and tensor polarized deuteron $\mathrm{T}_{10}{ }^{\text {circ }}, \mathrm{T}_{11}{ }^{\text {circ }}, \mathrm{T}_{21}{ }^{\text {circ }}$ and $\mathrm{T}_{22}{ }^{\text {circ }}$. We found that for charged and neutral pion channels the vector asymmetry $\mathrm{T}_{10}{ }^{\text {circ }}$ is quite sizable in the forward and backward directions. This asymmetry is of great interest, because it determines the GerasimovDrell-Hearn sum rule ${ }^{[16]}$ which planned to be tested experimentally at different laboratories. The other vector asymmetry $\mathrm{T}_{11}{ }^{\text {circ }}$ shows a rather different behavior. Considerably smaller are the tensor asymmetries $\mathrm{T}_{21}{ }^{\text {circ }}$ and $\mathrm{T}_{22}{ }^{\text {circ }}$, but they are a little more sensitive to final state interaction effects.

\section{References}

[1] Darwish, E.M., Arenhoevel, H. and Schwamb, M., Eur. Phys. J., A16: 111 (2003).

[2] Darwish, E.M., Arenhoevel, H. and Schwamb, M., Eur. Phys. J., A17: 513 (2003).

[3] Arenhoevel, H., Fix, A. and Schwamb, M., Phys. Rev. Lett. 93: 202301 (2004); Arenhoevel, H., Fix, A. and Schwamb, M., Proc. GDH 2004, Norfolk, Virginia, June 1-5, (2004).

[4] Arenhoevel, H. and Fix, A., Phys. Rev., C72: 064004 (2005); Fix, A. and Arenhoevel, H., Phys. Rev., C72: 064005 (2005).

[5] Levchuk, M.I., et al., Phys. Rev., C74: 014004 (2006).

[6] Darwish, E.M., et al., Phys. Rev., C76: 044005 (2007).

[7] Darwish, E.M., Nucl. Phys., A735: 200 (2004).

[8] Darwish, E.M., J. Phys., G31: 105 (2005).

[9] Darwish, E.M. and Salam, A., Nucl. Phys., A759: 170 (2005).

[10] Al-Thoyaib, S.S., Int. J. Mod. Phys., E16: 1773 (2007).

[11] Bjorken, J.D. and Drell, S.D., Relativistic Quantum Mechanics, McGraw-Hill, New York (1964).

[12] Drechsel, D., et al., Nucl. Phys., A645: 145 (1999); MAID Program, http://www.kph.unimainz.de/MAID/maid2003/.

[13] Haidenbauer, J. and Plessas, W., Phys. Rev., C30: 1822 (1984); Phys. Rev., C32: 1424 (1985).

[14] Nozawa, S., Blankleider, B. and Lee, T.-S.H., Nucl. Phys., A513: 459 (1990).

[15] Garcilazo, H. and Mizutani, T., $\pi N N$ Systems, World Scientific, Singapore (1990).

[16] Gerasimov, S.B., Sov. J. Nucl.. Phys., 2: 430 (1965); Drell, S.D. and Hearn, A.C., Phys. Rev. Lett., 16: 908 (1966). 


\section{ملاحظات الاستقطاب المزدوجة \\ لتفاعل الإنتاج الضوئي للبيون من الديونزون}

\section{سليمان صالح الذيبب}

قسم الفيزياء - كلبة العلوم - جامعة القصبم - المدلكة العربية السعودية

المستخلص. تم في هذا البحث دراسة ملاحظات الاستقطاب

المزدوجة لتفاعل الإنتاج الضوئي المتفكل من الديوترون باستخدام

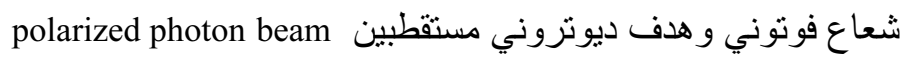
and polarized deuteron target

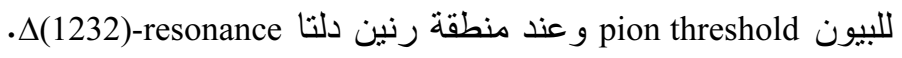
تم أيضاً دراسة ددى اعنماد هذه الملاحظات على التفاعلات التي

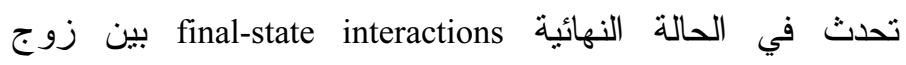
النيوكلونات nucleon-nucleon من ناحية، وبين البيون و النيوكلون

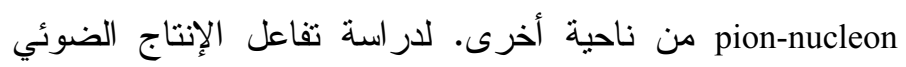

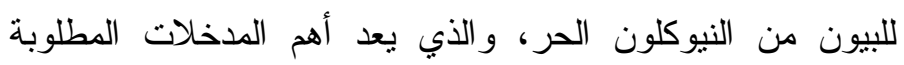

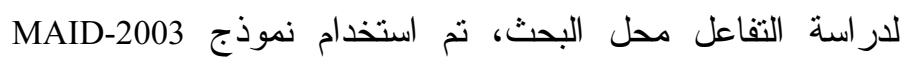
المعروف. بالنسبة لتفاعلات الحالة النهائية بين زوج النيوكلونات و البيون مع النيوكلون، تم استخدام نماذج جهد منفصلة separable

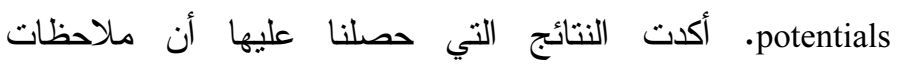
الاستقطاب المزدوجة للتفاعل محل الدراسة تعتمد بشدة على الثى تفاعلات الحالة النهائية وخاصة التفاعل بين زوجة التاعل النيوكلونات، حيث وجد أن تأثير التفاعل بين البيون والنيوكلون صغير جدأ. ولذلك يجب أخذ تفاعلات الحالة النهائية في الاعتبار عند تحليل نتائج ملاحظات الاستقطاب المزدوجة للتفاعل محل الدر اسة. 